\title{
SOOT EMISSION SIMULATIONS OF A SINGLE SECTOR MODEL COMBUSTOR USING INCOMPLETELY STIRRED REACTOR NETWORK MODELING
}

\author{
Savvas Gkantonas ${ }^{1, *}$, Jenna M. Foale ${ }^{1}$, Andrea Giusti ${ }^{2}$, Epaminondas Mastorakos ${ }^{1}$ \\ ${ }^{1}$ Department of Engineering, University of Cambridge, Cambridge, United Kingdom \\ ${ }^{2}$ Department of Mechanical Engineering, Imperial College London, London, United Kingdom
}

\begin{abstract}
The simulation of soot evolution is a problem of relevance for the development of low-emission aero-engine combustors. Apart from detailed CFD approaches, it is important to also develop models with modest computational cost so a large number of geometries can be explored, especially in view of the need to predict engine-out soot particle size distributions (PSDs) to meet future regulations. This paper presents an approach based on Incompletely Stirred Reactor Network (ISRN) modeling that simplifies calculations, allowing for the use of very complex chemistry and soot models. The method relies on a network of Incompletely Stirred Reactors (ISRs), which are inhomogeneous in terms of mixture fraction but characterized by homogeneous conditional averages, with the conditioning performed on the mixture fraction. The ISRN approach is demonstrated here for a single sector lean-burn model combustor operating on Jet-A1 fuel in pilotonly mode, for which detailed CFD and experimental data are available. Results show that reasonable accuracy is obtained at a significantly reduced computational cost. Real fuel chemistry and a detailed physicochemical sectional soot model are consequently employed to investigate the sensitivity of ISRN predictions to the chemical mechanism chosen and to provide an estimate of the soot particle size distribution at the combustor exit.
\end{abstract}

Keywords: Stirred Reactors, Aero-Engine Combustors, Kerosene, Soot, Particle Size Distribution

\section{NOMENCLATURE}

A area

D molecular diffusivity

$D_{t} \quad$ turbulent diffusivity

$F \quad$ stream index

$\dot{m} \quad$ mass flow rate

$N \quad$ scalar dissipation rate

$P_{\eta} \quad$ mixture fraction probability density function (PDF)

$Q_{\alpha} \quad$ conditionally-averaged mass fraction of species $\alpha$

$S c_{t} \quad$ turbulent Schmidt number

\footnotetext{
*Corresponding author: sg834@cam.ac.uk
}

$t \quad$ time

$\vec{u} \quad$ velocity vector

$V \quad$ volume

$Y_{\alpha} \quad$ mass fraction of species $\alpha$

$\eta \quad$ sample space variable of mixture fraction

$\xi \quad$ mixture fraction

$\rho \quad$ mass density

$\tau \quad$ residence time

$\dot{\omega}_{\alpha} \quad$ chemical source term of species $\alpha$

$\vec{\nabla} \quad$ gradient

$\vec{\nabla}$. divergence

- conventional mean

- Favre average

** $\quad$ core volume-averaged

$X_{\eta} \quad$ conditional scalar at particular $\eta$ value

$X_{\eta_{s t}} \quad \mathrm{X}$ defined at stoichiometry

$(\cdot)_{i} \quad$ stream indicator

$\langle\cdot \mid \eta\rangle \quad$ conditional Favre average

\section{INTRODUCTION}

The prediction of soot emissions and control of the particle sizes emitted from aero-engine combustors is of vital importance if next-generation low-emission engines are to mitigate the effects of particulate matter to human health and the environment [1]. However, the nature of soot evolution in turbulent flow poses significant numerical challenges. Apart from an accurate knowledge of turbulent mixing and finite-rate chemistry effects [2], reliable predictions of particulate emissions necessitate the use of complex soot models able to describe the underlying evolution processes [3] and the morphology of particles [4]. Predictions are known to be very sensitive to the choice of soot model $[5,6]$; therefore, sensitivity analysis and comparison between soot models are necessary to gain more insights into soot formation and oxidation. Nevertheless, recently developed comprehensive soot models [7-11] cannot easily be used in conjunction with highfidelity CFD simulations to perform parametric studies during the design phase of combustors, given their high dimensionality and 
computational cost. This becomes even more evident in jet fuel combustion, where detailed chemical schemes with additional Polycyclic Aromatic Hydrocarbons (PAH) routes (e.g., [9, 12]) also demand high computational requirements since a wide range of intermediate products and soot precursors should be considered. As a result, there is motivation to develop computationally inexpensive methods that simplify calculations with very complex chemistry and soot models, but also ensure the prediction of soot emissions within a reasonable degree of accuracy in both magnitude and trend.

In this direction, this study introduces a new approach utilizing Incompletely Stirred Reactor Network (ISRN) modeling to perform kinetic post-processing of CFD simulations. Due to its simplicity, this methodology allows for the use of comprehensive soot models with modest computational cost, while retaining approximate turbulence-chemistry interaction effects on the sooting flame structure. The Incompletely Stirred Reactor (ISR) model, developed by Bilger and coworkers [13-16], is a generalization of the Perfectly Stirred Reactor. Its derivation is based on the Conditional Moment Closure (CMC) method for turbulent reacting flows [17] and can be viewed as a zero-dimensional (or spatially-integrated) approximation of the full multi-dimensional CMC equation. An ISR is defined as a region of the flow, within which the flow and mixture fraction are inhomogeneous, but conditional averages of reacting scalars, conditioned on the mixture fraction, are uniform. In the limit of strongly recirculating flow, far from extinction, ignition, or other highly-transient phenomena, the latter assumption allows for the use of simple ordinary differential equations in mixture fraction space to model a combustor in its entirety, hence reducing the total computational cost. The approach was initially developed for non-premixed combustion but can be extended to multi-mode combustion following the newest developments of the CMC method [18].

The ISR method has been applied to experimental combustors $[15,19]$ and has demonstrated acceptable levels of accuracy, mostly limited by the fidelity of the mixing field, the appropriate choice of chemical mechanism and the satisfaction of the ISR conditions. Notwithstanding that the ISR conditions are generally achieved in the primary zone of a highly efficient aero-engine combustor, the ISR method may only be applied as a rough approximation for the prediction of soot emissions. As discussed in Refs. [11, 20, 21], conditional quantities related to soot evolution show strong spatial dependence. Therefore, it is necessary to extend the approach to a network of ISRs spanning the whole combustor under investigation. The combustor can then be partitioned in separate soot production and oxidation regions that exhibit different degrees of micro-mixing rates and residence times. In the context of kinetic post-processing, the ISRN equations can be decoupled from the calculation of the flow and mixing fields, consistently with other methods using ideal reactors (e.g., [22]). Unlike ideal reactors, however, unmixedness can be directly taken into account via the scalar dissipation rate and the mixture fraction probability density function. Their quantification is performed through averaging of the mixing field extracted from a reference CFD simulation, which could be of lower detail concerning the combustion and pollutant chemistry hence allowing significant computation time savings.
The main objective of this study is to investigate the ability of the ISRN approach to describe soot evolution in an aero-engine combustor. For this purpose, we apply the ISRN method to a model single sector combustor operated on Jet-A1 fuel for which experiments and detailed CFD data are available [23]. The model combustor replicates the conditions of a lean-burn engine at part load, where the injectors are operated in pilot-only mode. The resulting flame shows features typically associated with the diffusion combustion regime, which may give rise to non-negligible levels of soot. Pilot-only soot emissions are critical in the design stage of a lean-burn engine, so it is essential to develop a capability of predicting the soot particle size distribution (PSD). In this direction, we couple the recently developed hybrid chemistry (HyChem) approach for kerosene combustion [24, 25] with a reaction subset for molecular growth up to pyrene and a detailed physicochemical sectional soot model initially developed for ethylene [10, 11]. This allows for a demonstration of the ISRN potential in handling complex chemical mechanisms and soot models, but it also allows for an estimation of the soot PSD at the combustor exit. The soot PSD is generally a quantity of importance but very difficult to predict and reported only for laminar flame computations so far in the open literature.

The specific objectives of the work are to (i) assess the performance of the ISRN method compared to the detailed CFD data and the experimental measurements; (ii) analyze the effect of gasand soot phase chemistry on ISRN soot predictions; (iii) provide an estimate of the soot PSD in this combustor by employing a soot sectional model. This paper is structured as follows. The derivation of the ISRN equations is first introduced, followed by the description of the solution strategy and the models used in the computations. This is followed by a brief description of the investigated combustor and the reacting field obtained by the reference CFD simulation. Results and key conclusions close the paper.

\section{THE ISRN APPROACH}

\subsection{Mathematical model}

An ISR is considered to be a volume $V$ within which conditional averages of reacting scalars, conditioned on the mixture fraction, are independent of position and time. In contrast to a perfectly-stirred reactor, which has uniform composition, this allows an ISR to have mixture fraction inhomogeneities. Various versions of the ISR governing equations have appeared in the literature [13-16]. Here we extend the formulation given by Klimenko and Bilger [17] and derive the governing equations for a network of ISR reactors with an arbitrary number of inlet/outlet streams having non-uniform properties. The equations are derived from the CMC equation assuming high Reynolds number and negligible differential diffusion [17]:

$$
\begin{array}{r}
\frac{\partial \bar{\rho} Q_{\alpha} \widetilde{P}_{\eta}}{\partial t}+\vec{\nabla} \cdot\left(\bar{\rho}\left\langle\vec{u} Y_{\alpha} \mid \eta\right\rangle \widetilde{P}_{\eta}\right)=-\frac{\partial^{2} \bar{\rho}\langle N \mid \eta\rangle \widetilde{P}_{\eta}}{\partial \eta^{2}} Q_{\alpha} \\
+\bar{\rho}\langle N \mid \eta\rangle \widetilde{P}_{\eta} \frac{\partial^{2} Q_{\alpha}}{\partial \eta^{2}}+\bar{\rho}\left\langle\dot{\omega}_{\alpha} \mid \eta\right\rangle \widetilde{P}_{\eta}
\end{array}
$$

where $\eta$ is the sample space variable of the mixture fraction, $\xi, Q_{\alpha} \equiv\left\langle Y_{\alpha} \mid \eta\right\rangle$ is the conditional Favre-averaged mass fraction 
of a generic species, $\widetilde{P}_{\eta}$ the Favre-averaged probability density function (PDF) and $N \equiv D \vec{\nabla} \xi \cdot \vec{\nabla} \xi$ the scalar dissipation rate.

Considering statistically stationary flow, integration of Eq. (1) over the core volume and application of the flux divergence theorem to the LHS leads to:

$$
\begin{array}{r}
\oint_{A} \bar{\rho}\left\langle\vec{u} Y_{\alpha} \mid \eta\right\rangle \widetilde{P}_{\eta} d \vec{A}=-\int_{V}\left(\frac{\partial^{2} \bar{\rho}\langle N \mid \eta\rangle \widetilde{P}_{\eta}}{\partial \eta^{2}} Q_{\alpha}\right) d V \\
+\int_{V}\left(\bar{\rho}\langle N \mid \eta\rangle \widetilde{P}_{\eta} \frac{\partial^{2} Q_{\alpha}}{\partial \eta^{2}}+\bar{\rho}\left\langle\dot{\omega}_{\alpha} \mid \eta\right\rangle \widetilde{P}_{\eta}\right) d V
\end{array}
$$

In ISR theory [17], conditional correlations between reacting scalars and velocity are neglected, but they are retained here, as they may be of the same magnitude with convective fluxes. These are closed with a diffusion approximation [17], assuming a turbulent diffusivity $D_{t}=\mu_{t} /\left(\bar{\rho} S c_{t}\right)$ with $S c_{t}=0.4$ equal for all scalars. By definition, conditional reactive scalar statistics are considered uniform inside an ISR core. Hence, they can be moved out of the integral on the RHS of Eq. (2). Moreover, conditional statistics are only a function of mixture fraction. Therefore, their partial derivatives may be transformed into ordinary ones. The governing equation can be written in the following discrete form:

$$
\begin{array}{r}
\sum_{i=1}^{F^{\text {out }}}\left(\dot{m} \widetilde{P}_{\eta} Q_{\alpha}\right)_{i}-\sum_{j=1}^{F^{i n}}\left(\dot{m} \widetilde{P}_{\eta} Q_{\alpha}\right)_{j}+\sum_{i=1}^{F}\left(\bar{\rho} D_{t}\left(\vec{\nabla} Q_{\alpha}\right) \widetilde{P}_{\eta} \vec{A}\right)_{i} \\
=V \rho^{* *}\left(P_{\eta}^{* *}\left(N_{\eta}^{* *} \frac{d^{2} Q_{\alpha}}{d \eta^{2}}+\dot{\omega}_{\alpha} \mid \eta\right)-Q_{\alpha} \frac{d^{2} N_{\eta}^{* *} P_{\eta}^{* *}}{d \eta^{2}}\right)
\end{array}
$$

where the core volume-averaged mass density, $\rho^{* *}$, mixture fraction PDF, $P_{\eta}^{* *}$, and scalar dissipation rate, $N_{\eta}^{* *}$ have been introduced. The core volume-averaged quantities are given by:

$\rho^{* *} \equiv \frac{\int_{V} \bar{\rho} d V}{V} ; \quad P_{\eta}^{* *} \equiv \frac{\int_{V} \bar{\rho} \widetilde{P}_{\eta} d V}{V \rho^{* *}} ; \quad N_{\eta}^{* *} \equiv \frac{\int_{V} \bar{\rho}\langle N \mid \eta\rangle P_{\eta} d V}{V \rho^{* *} P_{\eta}^{* *}}$

Note the last term on the RHS of Eq. (3). To close this term, the transport equation for the mixture fraction PDF may be utilized [17]. Given a statistically stationary flow and neglecting molecular fluxes, the PDF equation becomes:

$$
\begin{array}{r}
\vec{\nabla} \cdot\left(\bar{\rho}\langle\vec{u} \mid \eta\rangle \widetilde{P}_{\eta}\right)=-\frac{\partial^{2} \bar{\rho}\langle N \mid \eta\rangle \widetilde{P}_{\eta}}{\partial \eta^{2}} \\
\Rightarrow \quad \sum_{i=1}^{F^{\text {out }}}\left(\dot{m} \widetilde{P}_{\eta}\right)_{i}-\sum_{j=1}^{F^{i n}}\left(\dot{m} \widetilde{P}_{\eta}\right)_{j}=-V \rho^{* *} \frac{d^{2} N_{\eta}^{* *} P_{\eta}^{* *}}{d \eta^{2}}
\end{array}
$$

where the flux divergence theorem and the definitions of Eq. (4) are employed. Consistently with the stirred reactor concept, the conditional quantities exiting the ISR may be taken equal to conditional quantities in the core, hence $\left(Q_{\alpha}\right)_{i, \text { out }}=Q_{\alpha}, \forall i=$ $\left[1, F^{\text {out }}\right]$. However, it is not necessary to assume that the outlet streams PDF are identical to the core [16]. Finally, the combination of Eqs. (3)-(5) leads to the governing equation of an ISRN element:

$$
\begin{aligned}
\sum_{j=1}^{F^{i n}}\left(\dot{m} \widetilde{P}_{\eta}\right)_{j}\left[Q_{\alpha}-\right. & \left.\left(Q_{\alpha}\right)_{j}\right]+\sum_{i=1}^{F}\left(\bar{\rho} D_{t}\left(\vec{\nabla} Q_{\alpha}\right) \widetilde{P}_{\eta} \vec{A}\right)_{i} \\
& =V \rho^{* *} P_{\eta}^{* *}\left(N_{\eta}^{* *} \frac{d^{2} Q_{\alpha}}{d \eta^{2}}+\left\langle\dot{\omega}_{\alpha} \mid \eta\right\rangle\right)
\end{aligned}
$$

A similar equation, without a chemical source term, is solved for the conditional mean enthalpy. Here, it is important to note that Eq. 6 is identical to the governing equation of Ref. [17] when the second term on the LHS is removed, and a single ISR reactor is employed having a single inlet and outlet stream.

\subsection{Solution strategy}

The ISRN approach starts with the creation of a standard computational grid and the calculation of the average reacting flow field using CFD. The modeling approach for the reference CFD simulation can vary given the availability of resources, but care must be taken for each specific application, so the underlying mixing field is well captured. Existing methods may include, for example, the use of RANS or LES coupled with the flamelet approach, CMC, transported PDF, or the Eddy Dissipation concept [26].

The ISRN equations are then solved using an in-house unstructured finite volume code, initially developed for CMC modeling (see $[10,23,27,28]$ and references therein). The ISRN is reconstructed around the grid of a reference CFD simulation by exploiting the topology of the CFD faces. After providing the ISR centroids, the reactors volumes are obtained through agglomeration of CFD cells based on the minimum distance between cells and centroids. This allows for arbitrary ISR spacing and to directly conserve the mass fluxes computed at the CFD resolution, also facilitating data transfer between solvers. In contrast with ideal reactor network approaches (e.g., [22, 29]), clustering procedures are not required to identify chemically and physically homogeneous zones since ISRs are inhomogeneous in terms of their flow and mixture fraction fields. The current implementation also permits the use of parallel evaluation of reactors, hence speeding up computations.

The evaluation of core volume-averaged quantities and areaaveraged fluxes requires knowledge of the mean flow and mixing fields. In this work, these quantities are extracted from a LES-CMC simulation, which will be described in detail later. CFD derived quantities are either evaluated at the faces of ISR reactors to calculate LHS terms of Eq. (6), such as $\dot{m} \widetilde{P}_{\eta}$ and $D_{t}$, or used during core volume-averaging. The procedure is very similar to the one followed every time-step in existing CMC codes (e.g., [27]). After the end of the calculation, unconditional quantities may be derived through the integration of the mixture fraction PDF. Here, unconditional values are calculated at the CFD grid after applying an inverse square distance interpolation over neighbor ISR reactors. The PDF is modeled here with a presumed $\beta$-function computed from the mixture fraction, $\widetilde{\xi}$, and the mixture fraction variance, $\widetilde{\xi^{\prime \prime 2}}$, of each CFD cell. In the case of singularities at the $\eta$-space boundaries, the $\beta$-function is replaced by a delta function. 
The core volume-averaged scalar dissipation rate is obtained through Eq. (4). To calculate the conditional scalar dissipation rate at the CFD level, the Amplitude Mapping Closure (AMC) model [30] is employed, i.e., $\langle N \mid \eta\rangle=N_{0} G_{\eta}$ where $G_{\eta}$ and $N_{0}$ are $G_{\eta}=\exp \left(-2\left[\operatorname{erf}^{-1}(2 \eta-1)\right]^{2}\right)$ and $N_{0}=\widetilde{N} / \int_{0}^{1} \widetilde{P_{\eta}} G_{\eta} d \eta$. The average scalar dissipation rate, $\widetilde{N}$, is readily available from the CFD simulation. It is important to note that in contrast with existing ISR models [13-16], here, the core volume-averaged scalar dissipation rate is not based on the double integration of Eq. (5), raising issues of consistency with the PDF transport equation. Nonetheless, it has been observed that in a multi-dimensional system such as an ISRN, the integration may result in negative values of the scalar dissipation rate being obtained through machine precision errors when the PDF tends to zero [17]. Here, we preferred a more robust approach in evaluating the conditional scalar dissipation rate, which is also consistent with state-of-theart CMC approaches (e.g., [28]).

An operator splitting technique is implemented for the solution of the ISRN equations which are marched in time with a constant time-step, $\delta t=2 \cdot 10^{-6} \mathrm{~s}$, until convergence is reached. Transport in physical space, i.e., LHS terms of Eq. (6), is solved first, followed by diffusion in mixture fraction space and the chemical source term integration. The chemical source term is closed with a first-order approximation. More details regarding the chemical source term integration will be provided in the next section as they differ depending on the type of soot model. A description of the soot models and the chemical mechanisms used in this work is also given in Section 3. A first-order upwind scheme is used for the evaluation of conditional gradients, whereas the diffusion term in mixture fraction space is discretized with a second-order scheme. The inert mixing solution is imposed at the inlets, and reactors are initialized with a fully-burning solution. Both walls and outlets are modeled with a zero-gradient condition. A constant pressure condition is used for all reactors. Mixture fraction space is discretized using 61 bins clustered around the stoichiometric mixture fraction $\left(\eta_{s t} \approx 0.063\right)$. Consistently with the reference CFD simulation [23], pure air at $T_{30}$ is imposed at $\eta=0$, whereas $\eta=1$ corresponds to pure vaporized Jet-A1 fuel at saturation conditions. As will be described in Section $4, T_{30}$ corresponds to a reference air temperature in the investigated rig.

\section{CHEMISTRY AND SOOT MODELING}

Provided a reference mixing field and an established solution strategy, the ISRN can be used to evaluate the sensitivity of soot predictions to the choice of gas-phase chemistry and soot modeling. In the following, the chemical mechanisms and soot models implemented in this work are discussed. To better understand the sooting tendency differences between the various models, preliminary computations with the OD-CMC approach are also provided in Section 3.3.

\subsection{Gaseous phase}

3.1.1 Surrogate dodecane (HU) mechanism. Here, kerosene is modeled as a single-component surrogate with dodecane as the main species, and a detailed chemical mechanism with 38 species (based on the mechanism of Ref. [31] and specifically calibrated for kerosene combustion) is employed. Acetylene $\left(\mathrm{C}_{2} \mathrm{H}_{2}\right)$ is part of the intermediate pyrolysis products, which is required for coupling with the two-equation soot model, as will be discussed later. The same mechanism was employed in the reference CFD simulation [23], so it was also chosen here to directly assess the performance of the ISRN compared to the detailed CFD. In the rest of this paper, this chemical mechanism will be referred to as HU (Heidelberg University) scheme.

3.1.2 HyChem mechanism. In order to have access to additional precursors for investigations with the physicochemical sectional soot model employed here, a more detailed model for kerosene fuel pyrolysis was deemed necessary. Recently, a hybrid chemistry (HyChem) approach [24, 25] has been proposed for the high-temperature combustion chemistry of jet fuel, as an alternative to the surrogate fuel approach (e.g., [32]). The HyChem mechanism was developed using experimental shock-tube and flow-reactor studies on speciation time profile data. The mechanism models kerosene as a single-component fuel and lumps the fuel pyrolysis into seven semi-global reaction steps involving nine to twelve species per reaction equation. The primary pyrolysis products for kerosene in the mechanism are: $\mathrm{H}, \mathrm{CH}_{4}, \mathrm{C}_{2} \mathrm{H}_{4}$, $\mathrm{C}_{3} \mathrm{H}_{6}, 1-\mathrm{C}_{4} \mathrm{H}_{8}$ (1-butene), $\mathrm{i}-\mathrm{C}_{4} \mathrm{H}_{8}$ (iso-butene), $\mathrm{C}_{6} \mathrm{H}_{6}$ (benzene), $\mathrm{C}_{6} \mathrm{H}_{5} \mathrm{CH}_{3}$ (toluene), and the methyl radical $\mathrm{CH}_{3}$. The oxidation of the pyrolysis products is consequently based on the USC Mech-II mechanism [33] with updated reactions for i- $\mathrm{C}_{4} \mathrm{H}_{8}$.

The HyChem mechanism includes aromatic hydrocarbons, such as benzene and toluene. However, larger Polycyclic Aromatic Hydrocarbons (PAH) species are not considered, but they are typically required by comprehensive soot models, like the Napoli sectional (NAPS) soot model employed in this work. Therefore, the HyChem combustion chemistry is combined here with additional PAH growth routes, extracted from a detailed mechanism calibrated for ethylene [10,11], which is also the base gas-phase scheme for the NAPS soot model. These reactions consider the sequential addition of acetylene molecules and the self-combination of resonantly stabilized radicals to account for the molecular growth of aromatic cycles up to pyrene (A4). A similar approach was followed in Ref. [9], where the HyChem model was combined with the KAUST PAH mechanism 2 [34] to study soot evolution in a jet fuel laminar co-flow flame. Overall, the combined HyChem and PAH chemistry consists of 886 reactions and 136 species, whereas the original HyChem approach has 843 reactions and 119 species. As shown in Appendix A, the main fuel pyrolysis and oxidation products are not modified substantially by the additional PAH routes. Therefore, for brevity, the combined scheme will be later referred to as HyChem mechanism.

\subsection{Soot models}

3.2.1 Two-equation model (2EQN). For the first part of this study, simulations are based on a semi-empirical two-equation soot model [35, 36], following the strategy in Ref. [23]. This model is based on the transport of two additional quantities, namely the soot mass fraction $\left(Y_{S}\right)$ and the soot number density $\left(N_{s}\right)$. Equations conditioned on the mixture fraction are solved for both of these quantities, similar to Eq. 6. The soot model accounts for nucleation and surface growth via acetylene 
$\left(\mathrm{C}_{2} \mathrm{H}_{2}\right)$, surface oxidation via $\mathrm{OH}$ and $\mathrm{O}_{2}$, but also coagulation by employing a similarity solution for the coalescence of spherical particles in the free molecular regime. This approach employs a mono-disperse approximation for the particle phase but ensures a varying mean mass diameter in physical space, directly affecting surface reactions. Although simple, this model, coupled with LES-CMC, was able to give good predictions of soot location in the investigated model combustor [23].

As far as the implementation aspect of the chemistry integration is concerned, chemistry integration has been split into two steps. First, the gas-phase chemistry is solved using the SpeedCHEM solver, which significantly speeds up the computation by exploiting a sparse analytical Jacobian formulation and tabulation of temperature-dependent properties [37]. In this work, both the HU scheme and the HyChem approach will be evaluated. This is followed by the integration of the soot chemistry, performed using the VODPK solver [38], which includes the soot mass fraction and number density but also all the gas-phase species involved in the soot chemistry. The error introduced from the latter split was evaluated by means of OD-CMC simulations [28] through comparison with a fully-coupled solution obtained with the method of lines. The total difference was found to be negligible for a range of scalar dissipation rates, thus allowing to speed up the computation without any accuracy penalty, as also discussed in Ref. [21] for ethylene combustion.

3.2.2 Napoli sectional model (NAPS). In this work, computations are also based on a detailed physicochemical sectional model that has been developed and extensively tested by D'Anna and co-workers (see [39] and references therein). This model has been demonstrated to successfully predict soot formation for various simple fuels and flame configurations while supporting the latest experimental evidence of soot evolution in laminar flames $[1,3,40]$. A simplified version of this approach for ethylene, also called the NAPS soot model, has been recently presented in Refs. $[10,11]$ and will be employed here. The NAPS model has been primarily validated for C1-C2 fuels [39] while here it was used for kerosene fuel, which is a large extrapolation. To ensure that soot related quantities remain realistic, reaction rate constants affecting soot surface growth via hydrogen-abstractioncarbon-addition (HACA) routes, have been adjusted and reduced by a factor of 20 . This modification is similar to what is commonly done in laminar sooting flames, where the HACA growth parameter, often referred to as "parameter alpha", is adjusted to match the maximum soot volume fraction obtained by simulations with values originated from experiments (e.g., [9, 12]). We did not attempt any further validation or calibration of the model for kerosene fuel. This should be the subject of future work, and ISRN predictions should be revisited with soot models developed explicitly for kerosene. Consequently, the results reported here with the NAPS model are meant to be only of indicative value concerning the comparison between ISRN and LES-CMC and soot locations against experiment, and not as any validation of the soot predictions for kerosene. The use of the NAPS model is useful since it may provide an estimate of the soot PSD in the primary region and at the exit of the investigated model combustor. Besides, the coupling of the ISRN with such a comprehensive soot model demonstrates the ability of the ISRN approach to simplify calculations with very complex and computationally expensive chemistry.

The NAPS soot model relies on a gas-phase mechanism containing PAH species up to pyrene [10,11]. For this purpose, the HyChem approach was here extended with an additional subset of PAH growth reactions, as discussed in Section 3.1.2. All species with greater molecular mass are treated as lumped species and are divided into classes incorporated in the soot sectional model. Reactions for the lumped species and the gaseous-phase species involved in the soot model are treated as elementary reactions in accordance with the sectional approach. Particle size distribution is defined by a single range of sections (bins), each containing a nominal hydrocarbon species in order of increasing atomic mass. Carbon number ranges from 24 to $2 \times 10^{10}$, which is equivalent to a mean mass diameter, $D_{m m}$, range of $0.8-630 \mathrm{~nm}$ assuming a constant soot particle density $\rho_{s}=1800 \mathrm{~kg} / \mathrm{m}^{3}$. Twenty-two sections are used in a geometric series with a carbon number ratio of, at least, two between sections. For each carbon number $n_{C}$, a stable and a radical form, i.e., a stable lumped species missing one $\mathrm{H}$-atom, is used so that particles are classified both by their size and state. Other properties, such as hydrogen content, are extracted from the original version [39] of the model and are directly considered in the formulation of kinetic rates. All known physical and chemical pathways affecting soot evolution are included in the particle phase scheme, and reactions are based on a modified Arrhenius expression with an additional dependency on the carbon number. The reaction classes consider: (i) particle inception via aromatics dimerisation, (ii) surface growth via $\mathrm{C}_{2} \mathrm{H}_{2}$, (iii) condensation of aromatics, (iv) oxidation via $\mathrm{O}_{2}$ and $\mathrm{OH}$, (v) particle coagulation via both coalescence and agglomeration, as well as (vi) $\mathrm{O}_{2}$ oxidation-induced fragmentation. Overall, the lumped species mechanism contains 1763 reactions and 44 bins.

In contrast with the chemistry integration method for the 2EQN soot model, a single operator splitting step was chosen here for both the gaseous phase and soot chemistry. As discussed in Refs. $[10,11]$, a split between the gas- and soot phases chemistry might result in a significant accuracy penalty because of the presence of aromatics-based pathways.

\subsection{PRELIMINARY COMPUTATIONS}

Although the sooting flame structure in an ISR reactor (or a CMC computation) is a result of the local scalar dissipation rate and transport terms, simplified computations considering only micro-mixing and chemistry effects can be used to understand the sooting tendency differences between the various combinations of gas- and soot-phase models, summarized in Table 1. For this purpose, the so-called 0D-CMC approach (i.e., solution of Eq. 6 without LHS terms and $N_{\eta}^{* *}=N_{0} G_{\eta}$ with prescribed $N_{0}$ ), which is similar to a flamelet solution with unity Lewis number and an imposed scalar dissipation rate profile [28], is employed. The results are a useful reference for the remainder of the discussion.

Figure 1 shows the flame structure in $\eta$-space for $N_{0}=10 \mathrm{~s}^{-1}$ at the conditions used in the ISRN simulations (see Section 2.2). Firstly, a comparison of the HU-2EQN and HyChem-2EQN models shows that they are characterized by significantly different acetylene $\left(\mathrm{C}_{2} \mathrm{H}_{2}\right)$ mass fractions (by a factor of 2), directly affecting sooting tendency. Notably, the HU-2EQN model shows 
TABLE 1: CHEMICAL SCHEMES AND SOOT MODELS USED FOR SIMULATIONS

\begin{tabular}{lcc}
\hline Mechanism & Soot Model & Description \\
\hline HU & 2EQN & 38 species \& 174 reactions \\
HyChem & 2EQN & 119 species \& 843 reactions \\
HyChem & NAPS & 180 species \& 2649 reactions \\
\hline
\end{tabular}

higher values of soot than the HyChem-2EQN model (in terms of volume fraction, $f_{v}$, mass fraction, $Y_{s}$, and number density, $N_{s}$ ), linked to the higher levels of acetylene. This is especially important for soot evolution, as residence time effects and local micro-mixing are expected to increase the disparity between models further, even though the relative locations of soot related quantities (with respect to mixture fraction) are similar.

Moreover, results with the HyChem-NAPS model are also included in Fig. 1. It is found that the soot volume (mass) fraction is smaller compared to the 2EQN model cases. Notably, the maximum soot volume fraction obtained with HyChem-NAPS is smaller by a factor of 1.6 and 1.27 compared to HU-2EQN and HyChem-2EQN, whereas the maximum soot mass of the HyChem-NAPS is almost half compared to HU-2EQN and 1.8 times smaller than HyChem-2EQN. Despite changes in magnitude, the relative locations of soot volume (mass) fraction in $\eta$-space are again similar between models. Nevertheless, the NAPS model sensitivity to scalar dissipation rate and residence time is expected to be very different, due to the fundamental differences of NAPS with acetylene-based models. It is important to note that the comparisons above are only of indicative value, and should only be considered for the assessment of the ISRN predictions later. As described in Section 3.2.2, it was found necessary to adjust the HACA-based reaction rate constants for soot surface growth. This was to avoid high levels of soot but also soot leakage, caused by unphysically fast growth and possibly slow oxidation on the lean side of $\eta_{s t}$. The chemical composition and reactivity of soot particles generally vary between fuels; therefore soot models developed for simple fuels, such as the NAPS model here for ethylene, are generally not expected to perform similarly for real fuels. Typically, an empirical soot surface growth parameter is used (see e.g., $[9,12]$ ) to tune surface reactions, therefore a similar modification has been applied here by reducing the equivalent reactivity of particles subject to surface growth. It is also noteworthy that the particle size distribution is also affected. Here, nanoparticle formation is favored at scalar dissipation rate $N_{0}=10 \mathrm{l} / \mathrm{s}$, as inferred by the orders of magnitude difference between the total number density $\left(N_{s, t o t}\right)$ and the number density of larger soot particles $\left(N_{s, d>7 \mathrm{~nm}}\right)$ for the HyChem-NAPS model in Fig. 1d. The above should be considered during future developments of the HyChem-NAPS model for kerosene combustion, so a more accurate balance between soot formation and oxidation is established.

\section{INVESTIGATED BURNER AND CFD ANALYSIS}

The rig investigated in this paper [41], named "Big Optical Single Sector", is a single sector model combustor reproducing the main characteristics of a real engine. The rig allows operation

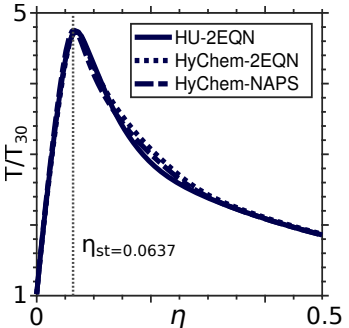

(a) Temperature

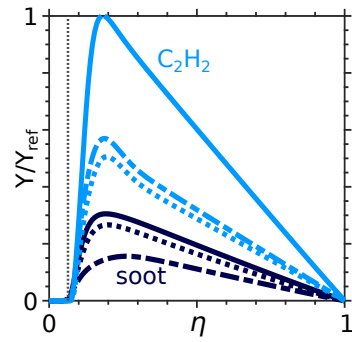

(c) Mass fractions

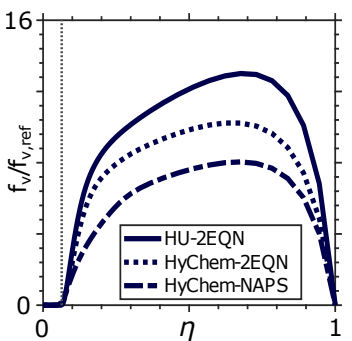

(b) Soot volume fraction

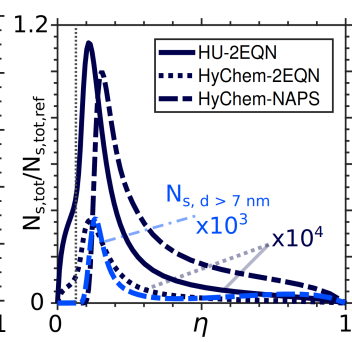

(d) Soot number density

\section{FIGURE 1: CONDITIONAL QUANTITIES IN MIXTURE FRACTION SPACE FROM OD-CMC COMPUTATIONS $\left(N_{0}=101 / \mathrm{s}\right)$. LEGENDS APPLY TO ALL QUANTITIES, INCLUDING THOSE IN (c).}

of original engine-size lean burners with pressures up to 20 bar and provides wide optical access to the primary zone of the combustor enabling the use of planar laser-based spectroscopic techniques. A detailed description of the rig can be found in Ref. [23], so only a brief summary is given in the following. Further details regarding the experimental rig can also be found in Refs. [41, 42].

Figure 2 shows the main characteristics and components of the test section [42]. The overall length of the combustor is about $250 \mathrm{~mm}$. The combustion chamber comprises a square section enclosure with dimensions $140 \times 140 \mathrm{~mm}$ immediately downstream of the injector exit. The optical segment (a) extends from the injector exit $40 \mathrm{~mm}$ downstream. The inner surface is protected by a starter film of $1 \mathrm{~mm}$ thickness [41], with adjustable mass flow and temperature. The downstream part of the rig is characterized by an effusion-cooled square transition piece (c) and an effusion-cooled converging section (e). For the conditions in the present work, the temperature of the film was set equal to the temperature, $\mathrm{T}_{30}$, of the air flowing through the injector with a mass flow rate of about $30 \%$ of the mass flow through the injector. The rig is operated with kerosene (Jet-A1) as fuel. The injector is based on a two-stage configuration with the main and pilot fuel injection achieved through concentric airblast atomizers (more details on the injector configuration can be found in Ref. [43]). The outer airblast atomizer represents the main fuel injection with the pilot injector located at the centre. The condition investigated here corresponds to a part-load condition where the injector is operated in pilot-only mode. The operating pressure is the one of a low-power pilot-only condition with an overall air-to-fuel ratio (including all the cooling flows) of about 125.

Available experimental data for this condition include planar laser-induced fluorescence of OH (OH-PLIF), phase Doppler anemometry (PDA), and laser-induced incandescence (LII) mea- 
surements for soot location. A schematic of the streamwise crosssection of the combustor with the observation windows explicitly used in the LII measurements is shown in Fig. 3. This is useful to illustrate the orientation of the LII region with respect to the combustor and the soot volume fraction predictions discussed later. Experimental results, as well as a detailed description of the experimental methods, can be found in the work by Giusti et al. [23]. In the same study, the part-load condition investigated here was simulated using the LES-CMC approach coupled with the HU-2EQN scheme (see Section 3). It was shown that LES-CMC is able to predict the main characteristics of the flame, showing a good agreement with the experiment in terms of flame shape, spray characteristics, and soot location. As a result, the combustor underlying mixing field is well captured, and the simulation data can be used to provide the ISRN with the mean velocity and mixing fields. In addition, the LES-CMC simulation can be used as a reference to assess the predictability of the ISRN approach. The LES-CMC approach is based on the time-resolved solution of the local flame structure in mixture fraction space and incorporates molecular mixing and turbulent transport effects. This allows for the reproduction of multi-scale turbulence-chemistry-soot interactions and to properly account for history and finite-rate chemistry effects on soot evolution in a spray flame. Consequently, LES-CMC is more than appropriate as a benchmark for the IRSN method.

The computational domain used in the reference LES-CMC simulation and the ISRN computations is shown in Fig. 4. The domain reproduces the geometry of the experimental rig, including the plenum upstream of the injector. Details regarding the numerical setup and the boundary conditions can be found in Ref. [23]. An example of the instantaneous flame shape predicted by LES-CMC is shown in Fig. 5 through an isosurface of the stoichiometric mixture fraction colored with temperature. The stoichiometric mixture fraction surface is almost attached to the injection location; however, the flame appears lifted-off, as revealed by the low temperature close to the injector exit. Figure 6 shows the average axial velocity, temperature, and mixing field extracted from the simulation. It is evident that the conditions inside the recirculation zone are favorable for soot formation and growth, given the presence of rich mixture fractions and hightemperature residence time (due to recirculation). Despite the high levels of mixture fraction close to injector exit (associated with strong fuel evaporation [23]), high RMS values of the mixture fraction are also observed indicating high scalar dissipation rates that generally lead to lower values of soot.

Once the mixing and flow fields from the LES-CMC simulation are averaged, the ISRN approach is then applied to the same flame. For this study, the burner is partitioned in 154 ISR reactors uniformly positioned along the cross-wise directions. A cut of the ISRN grid is shown in Fig. 7a. A coarser ISR spacing with 78 ISR reactors (see Fig. 7b) has also been employed as a reference for computational cost comparisons. Hereafter, the analysis will focus only on the fine ISR spacing with only key results being reported later for the coarser ISR spacing. It is important to note that ISRN predictions are generally sensitive to the choice of reactor spacing, but a full parametric analysis was out of the scope of this study. Instead, the main objective

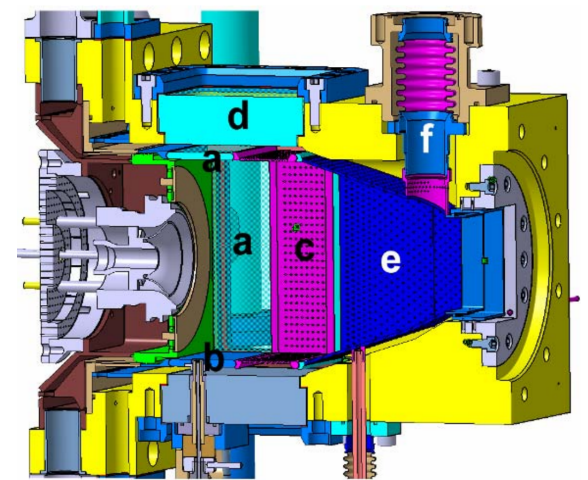

FIGURE 2: SCHEMATIC OF THE BIG OPTICAL SINGLE SECTOR RIG [42]

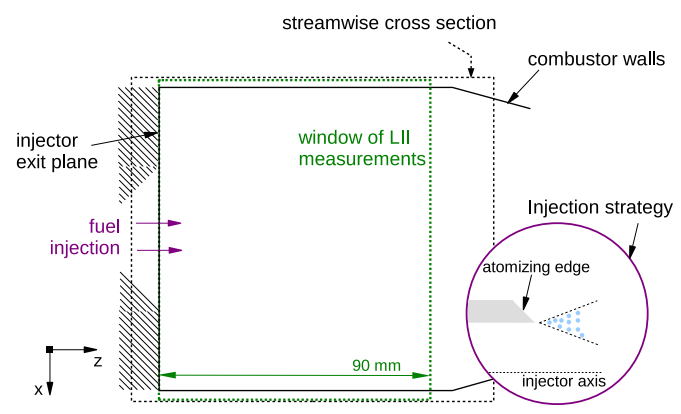

FIGURE 3: LII MEASUREMENT REGION AND INJECTION STRATEGY (ADAPTED FROM REF. [23])

is to provide a rough and quick estimate of the soot emission in this combustor. A more refined spacing, aiming to better resolve the gradients of conditional quantities in the soot production and oxidation regions, is expected to improve soot predictions due to the fact that transport effects in soot evolution are generally not negligible $[2,44]$. The key parameters controlling soot evolution within an ISRN are local equivalence ratio, residence time and scalar dissipation rate. Therefore, the choice of ISR spacing can be viewed as an attempt to capture the full extent of these parameters by adjusting the volume of ISRs and their relative location within the combustor. This rationale is further described in Appendix B and applied to the ISR spacings used in this study.

\section{RESULTS AND DISCUSSION}

Prior to the assessment of the ISRN in replicating soot evolution, the approach should be first evaluated for its ability to replicate the mean flame structure. Figure 8a shows the mean $\mathrm{OH}$ mass fraction and temperature fields in the primary reacting region extracted from the detailed LES-CMC simulation and the ISRN. The azimuthally-averaged fields are compared side-by-side for ease of evaluation. A comparison of LES-CMC with OHPLIF measurements is given in Ref. [23]. The overall agreement of LES-CMC with the experiment is satisfactory with the detailed simulation capturing the mean characteristics of the flame. The ISRN predictions are in very good agreement with LES-CMC, also demonstrating the capability of the ISRN approach in describing the reacting region. There are some differences in $\mathrm{OH}$ along the shear layer which may be due to the ISR spacing, a 


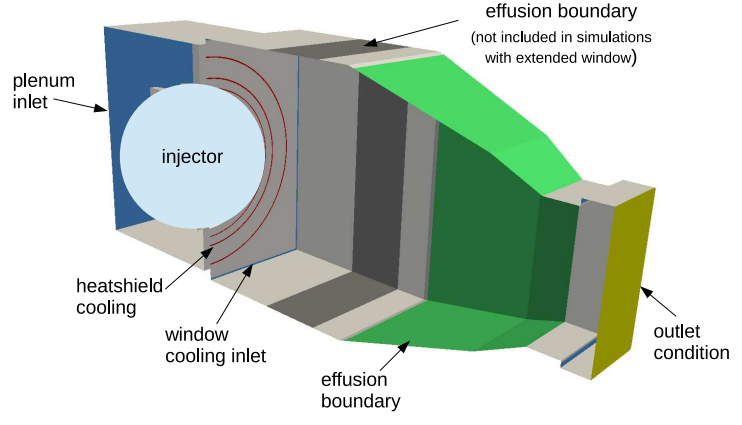

FIGURE 4: COMPUTATIONAL DOMAIN WITH RELEVANT BOUNDARY CONDITIONS [23]

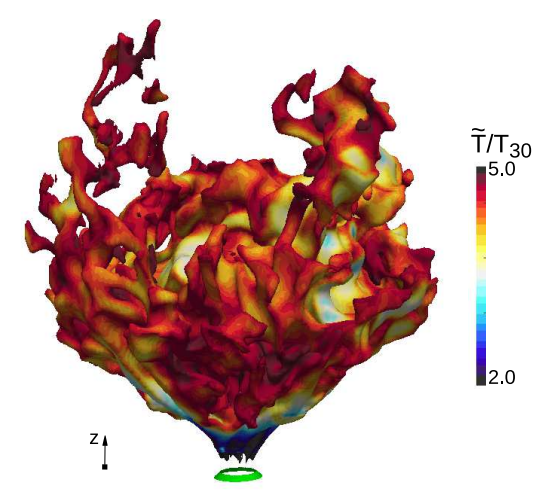

FIGURE 5: LES-CMC ISOSURFACE OF THE STOICHIOMETRIC MIXTURE FRACTION COLORED WITH TEMPERATURE [23]

topic discussed later in this section. These differences may be quantified via a cross-stream average at various heights downstream the injector, depicted in Fig. 8a. On average, the relative differences of the ISRN compared to LES-CMC are 40\%, $18 \%$, $-4.5 \%$ and $-32 \%$ at heights A to D. The comparison with temperature is shown in Fig. 8b. The difference in the peak is around $50 \mathrm{~K}$, whereas, on average, the corresponding relative difference at the various heights A-D are $-4.7 \%,-4.5 \%,-5.1 \%$ and $-6.5 \%$, respectively. The above show that the underlying reacting field is well represented and there is only a small mismatch between the two reacting fields due off-line approach used in the ISRN.

Figure 9 compares the experimental LII measurements of mean soot volume fraction with the predictions of the various computations. To facilitate the analysis, the difference between predictions and the measurements has been calculated at various heights downstream the injector, similar to Fig. 8. Here, a root mean square (rms) difference, $e_{r m s}$, is reported instead of a cross-stream average metric, allowing for an evaluation of both magnitude and relative spatial distribution. Considering the detailed LES-CMC results coupled with the HU-2EQN scheme, the simulation is in good agreement with the experiment in terms of soot location. The low value of soot volume fraction close to the injector exit and its increase in the downstream direction are predicted quite well. The experiments also show non-negligible amounts of soot along the combustor axis further downstream. It is also observed that regions of high soot content are found inside the recirculation zone (as inferred by Fig. 6). These features are
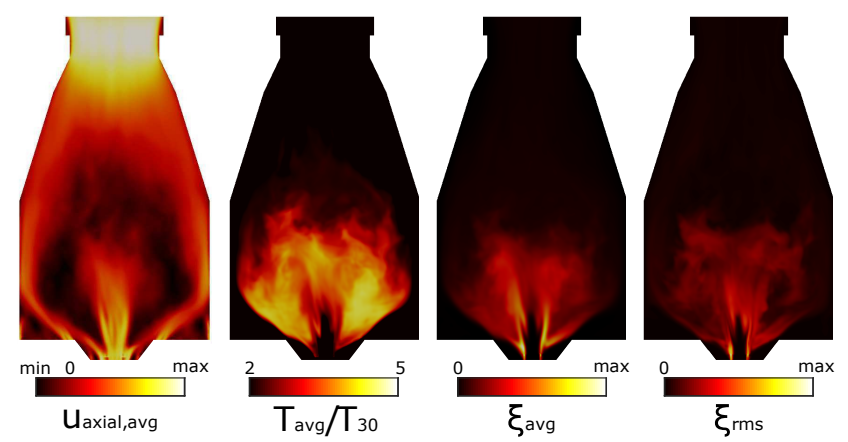

FIGURE 6: AVERAGE FIELDS FROM THE LES-CMC SIMULATION

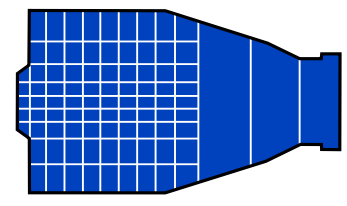

(a) Fine (154 ISRs)

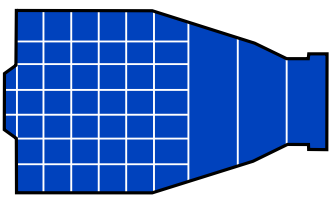

(b) Coarse (78 ISRs)
FIGURE 7: ISR SPACINGS FOR THE INVESTIGATED COMBUSTOR

captured by the LES-CMC simulation, but the predictions show a less uniform distribution, as shown by the $e_{r m s}$ evolution. As far as the absolute level of soot is concerned, the simulation generally over-predicts the experimental soot level [23] (note that the scale in the LII is logarithmic). Here, the predicted peak soot volume fraction is about 12 times higher than the maximum LII value recorded. This is an inherent feature of the $2 \mathrm{EQN}$ model and the simplified soot chemistry used here. Therefore investigations with more detailed soot models, explicitly calibrated for kerosene, are expected to lead to lower levels of soot as also observed for simpler fuels (e.g., [6]). As discussed in the following for ISRN predictions, the mean LES-CMC soot location is also expected to differ with a fundamentally different soot model, but further work is required to quantify these effects.

The LES-CMC results are then compared to the ISRN soot predictions with the HU-2EQN approach. This allows for a direct assessment of the ISRN ability to replicate soot evolution. The ISRN prediction matches reasonably well with the LES-CMC simulation. Soot is absent close to the injector exit, and a peak is observed at the same location as the LES-CMC. However, the extent of the mean soot location is qualitatively different. We hypothesize that the ISR spacing is not adequate to resolve the conditional gradients in the cross-wise directions, thus a better match can be obtained with the placement of more reactors. Computations with the coarser ISR spacing of Fig. 7b showed that the prediction of the mean soot volume fraction was indeed significantly affected, both in terms of magnitude and location. Notably, the $e_{r m s}$ values at heights A, B and D were no more than 5\% different as what obtained with the finer ISRN but at height C, $e_{r m s}$ was smaller by $25 \%$. By extrapolation, we might expect differences of same order when a much finer ISR spacing is employed. Nevertheless, a detailed sensitivity analysis was out of the scope of this study, and it should be the main subject of a future systematic investigation. The number of ISRs in the high-temperature 

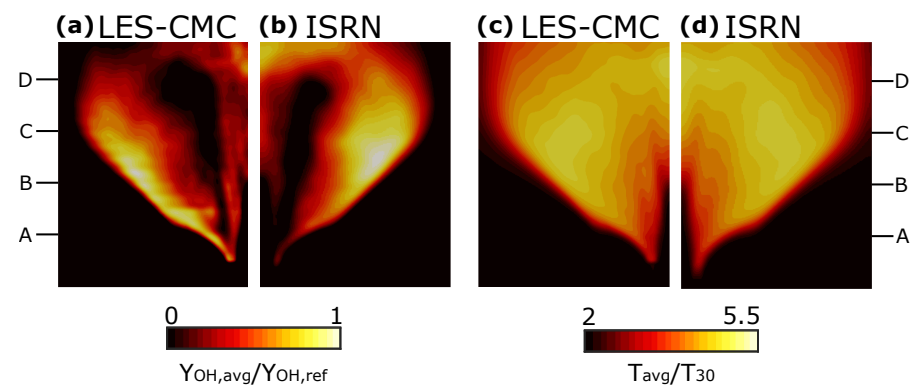

FIGURE 8: MEAN (a)-(b) OH MASS FRACTION AND (c)-(d) TEMPERATURE OF THE LES-CMC AND ISRN SIMULATIONS (HU-2EQN). A-D INDICATE THE HEIGHTS AT WHICH RELATIVE DIFFERENCES ARE REPORTED.

recirculation zone (where soot growth is favored) is an important parameter and should be carefully selected if a good estimation of the local scalar dissipation rate and the residence time within each ISR are to be ensured. However, a low number of ISRs with arbitrary spacing can still be useful as a rough estimate of soot emissions and inexpensive parametric analysis. Preliminary investigations in the ethylene Cambridge Rich-Quench-Lean (RQL) combustor [10, 11, 21, 45-47] also show the same qualitative differences between LES-CMC and ISRN predictions [48], possibly because of the coarse ISR spacing employed in the computations. Nevertheless, the same trend is not observed in a condition of the DLR ethylene combustor [49], which is also a target flame of the International Sooting Flame workshop. Recent investigations in this combustor show that the mean soot location can be very well captured by the ISRN, consistent with both detailed CFD and experimental data [50]. This is probably a result of the different flow patterns and range of scalar dissipation rates in this combustor compared to the Cambridge RQL burner and the big optical single sector combustor. As a result, further investigations are necessary to develop the ISRN method and associated solution strategies further. Some considerations regarding the evaluation of these strategies and the choice of ISR spacing are given in Appendix B.

As shown by the difference of the HU-2EQN predictions with ISRN and the LES-CMC, ISRN predictions generally show a higher soot volume fraction up to a factor of 7, stemming from the off-line approach used in the ISRN. Provided the mean flow and mixing fields, the evolution of conditional scalars, including soot volume fraction and temperature, is inherently different from LES-CMC since no two-way coupling with the reacting flow is attained and excursions of the scalar dissipation rate are not considered. However, the ISRN contains effects of micro-mixing and transport that are especially important during soot evolution; hence, an adequate balance between reaction-transport-diffusion is established. The latter is especially useful for parametric analyses and the search for soot tendency trends.

In this direction, the HyChem-2EQN and HyChem-NAPS (described in Section 3) are also employed and compared here to the ISRN predictions with the HU-2EQN scheme. Since all approaches share the same ISRN setup and underlying mixing field, differences between models are only attributed to the balance between soot formation and oxidation, also controlled by transport and local micro-mixing (see Eq. 6). Consistently with the 0D-CMC results of Fig. 1, HyChem-2EQN is characterized by a peak soot volume fraction similar to the HU-2EQN approach. However, the discrepancies in the acetylene levels observed with OD-CMC should generally lead to a different soot volume fraction field. The latter is a result of the different sensitivity of the underlying gas-phase schemes to the scalar dissipation rate and the corresponding residence time effects on soot evolution. Indeed, the ISRN predictions with HyChem-2EQN show a qualitatively different soot location than with HU-2EQN, as can also be inferred by the evolution of $e_{r m s}$. Less soot is evident in the region close to the injector, and the soot field appears less uniform with more abrupt transitions downstream (note the relative difference $e_{r m s}$ from height to height). As far as the peak soot volume fraction is concerned, results show a 48 times over-prediction compared to the experiment against an over-prediction of 64 with HU-2EQN. The latter observations are a typical example of the ISRN approach's usefulness in comparing gas-phase schemes and provide insights into soot evolution. The ISRN results here complement the preliminary computations with OD-CMC, and establish a link between the flame structure in mixture fraction space and quantities of interest in physical space.

Results with the HyChem-NAPS approach, which is even more complex and leads to predictions of the PSD, are now considered. The simulation shows qualitatively the same features with the 2EQN approaches regarding the location of the peak soot volume fraction, however, the HyChem-NAPS soot field extends further in the cross-wise directions, up to half the width of the combustion chamber, which is more consistent with the experiment and the LES-CMC. Notably, the variability of $e_{r m s}$ is also small, indicating the generally good match with the LII field, except for locations close to the injector where no soot is supposed to be present. Consistently with OD-CMC, the peak value of soot volume fraction is the smallest among all ISRN simulations and about 24 times higher than the experiment. Again, this demonstrates the need to develop the HyChem-NAPS approach specifically for kerosene fuel. Nevertheless, the ISRN data obtained with the HyChem-NAPS also allows for an estimate of the soot particle size distribution (PSD).

Figure 10 shows the evolution of the soot PSD within the core of ISRs located at different heights inside the primary region (schematically indicated in Fig. 9) and at the combustor exit. Firstly, it is found that ISRN with HyChem-NAPS predicts the presence of a wide range of particle sizes in the 1 to $100 \mathrm{~nm}$ range. A shift from a uni-modal to a bi-modal soot PSD shape is not evident in Points P1-P3 (or within the recirculation zone), consistent with experimental observations in turbulent flames [51, 52]. Nevertheless, the soot PSD at the exit clearly shows a bimodal character and the dominance of nanoparticles. The first peak is probably a result of coalescence of nanoparticles towards the exit of the combustor, where temperature is low and nucleation is not persistent. The second peak is mostly attributed to the balance of coagulation and oxidation/fragmentation reactions or larger particles observed at locations within the flame, such as Points P1-P3. Since soot evolution is strongly associated with history effects, the soot PSD at the exit is undoubtedly affected by soot predictions in the primary region. However, it is not 


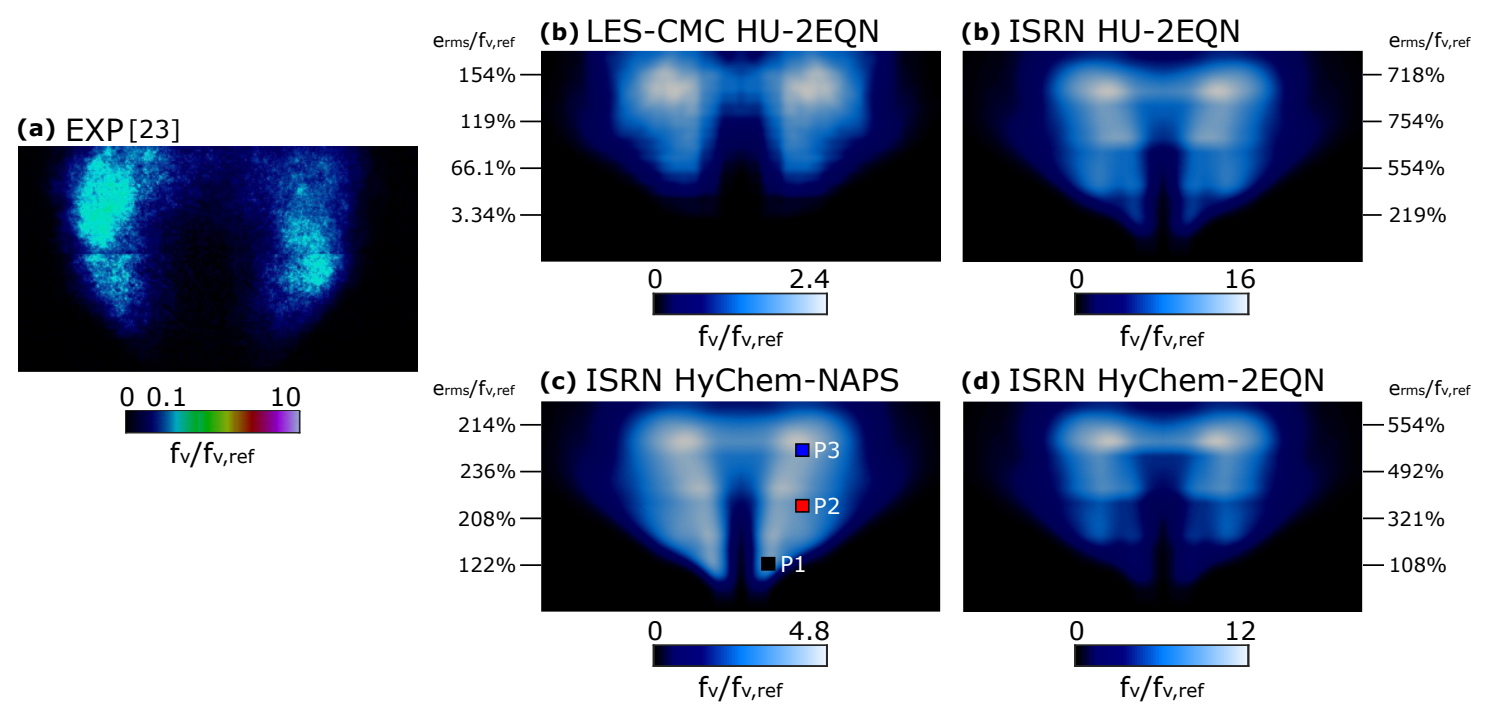

FIGURE 9: MEAN SOOT VOLUME FRACTION IN THE EXPERIMENT [23] AND NUMERICAL SIMULATIONS. THE RMS DIFFERENCE BETWEEN EXPERIMENT AND ISRN PREDICTIONS IS REPORTED AT HEIGHTS A (20 MM), B (40 MM), C (60 MM) AND D (80 MM) DOWNSTREAM THE INJECTOR. POINTS P1-P3 FOR SOOT PSD COMPARISONS ARE ALSO INDICATED.

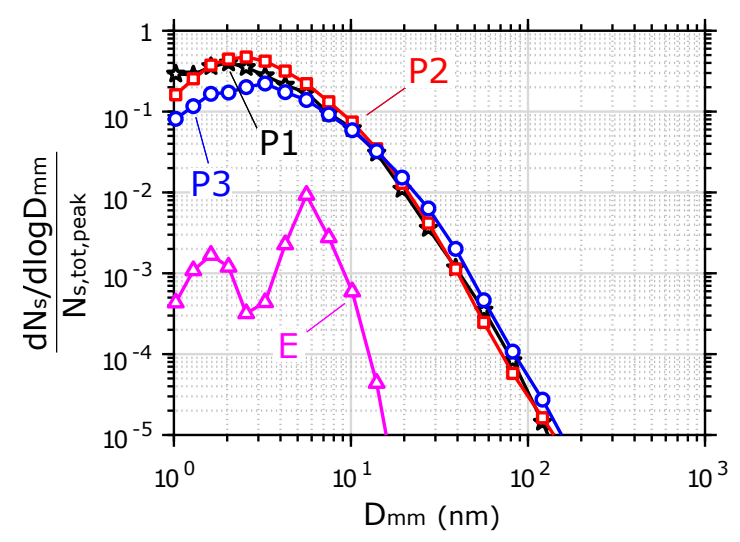

FIGURE 10: PREDICTIONS OF THE SOOT PARTICLE SIZE DISTRIBUTION AT POINTS P1-P3 (SEE FIG. 9 FOR LOCATIONS) AND AT THE COMBUSTOR-EXIT (E)

clear if uncertainties in the reactivity of particles, as discussed in Section 3.3, will have a strong effect on the PSD shape at the combustor exit. Future investigations with models explicitly calibrated for kerosene fuel could elucidate soot PSD evolution in this combustor.

As far as the implementation aspects of the ISRN are concerned, the method is computationally very efficient. As the mixing field is pre-calculated and only a subset of processes is solved at each time-step, the computational time is drastically minimized compared to a detailed CFD simulation. Notably, for the numerical setup presented here, more than $95 \%$ of the runtime is spent for the chemistry integration, which is comparable to laminar flamelet computations. Average runtimes with the various models used in this study are provided in Table 2 together with computational costs. A small number of reactors is typically adequate to provide an estimate of soot emission, as shown in Fig. 9. Further reduction of the number of ISRs can result in significant computational savings, as shown in Table 2. However, the use of a coarser ISRN may result in reduced accuracy as mentioned previously. Special attention is required when very coarse ISR spacings utilized, for which the rationale presented in Appendix B may prove useful to identify the optimum spacing. This will also be the focus of future investigations.

\section{TABLE 2: AVERAGE SIMULATION RUNTIMES}

Average runtimes for advancing the ISRN by $\delta t=2 \times 10^{-6} \mathrm{~s}$ and computational cost for $20 \mathrm{~ms}$ of simulation on one node (32

MPI processes) of an Intel Xeon Skylake supercomputer (Cambridge CSD3)

\begin{tabular}{lcccc}
\hline Approach & \multicolumn{2}{c}{$\begin{array}{c}\text { Average ISRN } \\
\text { runtime }(\mathrm{s})\end{array}$} & $\begin{array}{c}\text { Total computational } \\
\text { cost (CPUh) }\end{array}$ \\
\hline & Fine & Coarse & Fine & Coarse \\
HU-2EQN & 0.76 & 0.51 & 67.5 & 45.3 \\
HyChem-2EQN & 9.1 & 4.8 & 809 & 427 \\
HyChem-NAPS & 31.5 & 14.4 & 2,800 & 1,280 \\
\hline
\end{tabular}

Convergence with the ISRN is typically obtained after 10,000 iterations ( $20 \mathrm{~ms}$ for $\delta t=2 \times 10^{-6} \mathrm{~s}$ ), which corresponds to a total runtime of approximately 3.6 days for calculations with HyChemNAPS and the fine ISR spacing of Fig. 7a on one node (32 MPI processes) of an Intel Xeon Skylake supercomputer (Cambridge CSD3). The same performance can also be achieved in a modern multi-core workstation but is bounded by the available computer memory necessary to process the underlying CFD computation and the total number of scalars. Faster runtimes may be attained, as the total runtime scales inversely with the number of total MPI processes, but at a higher computational cost due to loss of efficiency in processor communication and load balancing. It is notable that even with the choice of one processor per ISR, e.g., for fine ISR spacing and the computationally expensive HyChemNAPS, the total computational cost can generally be lower by, 
at least, an order of magnitude compared to detailed LES with comprehensive soot models (e.g., [7, 11]). One processor per ISR would also result in a total runtime of about $18.2 \mathrm{~h}$, which is computationally very attractive compared to the amount time to complete a detailed CFD simulation (typically order of weeks or months).

In reality, it is hard to generalize as computational cost strongly depends on the type of simulation, the choice chemistry and models, the solvers, the numerical setup (e.g., number of CMC cells for LES-CMC) and the specifics of the system's architecture where a simulation is executed. Notably, the LESCMC simulation compared here, utilized about 100,000 CPUh, hence two to three orders of magnitude more than the ISRN costs reported in this study. However, solid conclusions regarding the total speed-up compared to LES-CMC cannot be drawn, as different solvers were employed for the chemistry integration [23]. The main benefit of the ISRN approach is that very complex chemical mechanisms and soot models can be investigated in turbulent flows with reasonably low computational requirements, available to most combustion engineers. Therefore, the ISRN approach could be employed for a priori assessment of these models before switching to detailed CFD methods.

\section{SUMMARY AND CONCLUSIONS}

A novel approach using Incompletely Stirred Reactor Network (ISRN) modeling for soot emission simulations in aeroengine combustors has been formulated. An Incompletely Stirred Reactor (ISR) is a region of the flow, within which the flow and mixture fraction are inhomogeneous, but conditional averages, conditioned on the mixture fraction, are uniform. A network of ISRs can then be deployed to separately capture soot production and oxidation regions exhibiting different degrees of micromixing rates and residence times. The network is constructed around the grid of a reference CFD simulation providing the ISRN with the average flow and mixing fields, hence simplifying calculations. The ISRN approach is demonstrated on a calculation of a single sector lean-burn model combustor operating on Jet-A1 fuel and in pilot-only mode, which corresponds to conditions of a lean-burn engine at part load. The ISRN predictions are compared with experimental and simulation data previously obtained with detailed CFD using surrogate dodecane chemistry and a two-equation model for soot. The comparison shows that reasonable accuracy can be produced while ensuring a significant reduction in computational costs.

Consequently, the ISRN method is tested with detailed fuel pyrolysis and oxidation chemistry, based on the hybrid chemistry (HyChem) approach. It is found that the choice of the underlying chemical mechanism and prediction of pyrolysis products have an impact on soot predictions, even when a two-equation soot model with acetylene-only routes is considered. From a soot precursor modeling perspective, it is therefore essential to test both detailed surrogate and HyChem-type models for real fuel combustion but also include large Polycyclic Aromatic Hydrocarbons (PAH) routes, necessary for comprehensive soot models. The latter is attempted for HyChem chemistry, which is here coupled with a reactions subset for molecular growth up to pyrene and a detailed physicochemical sectional soot model initially developed for ethylene combustion. This procedure allows for an estimate of the soot particle size distribution (PSD) at the exit, which would otherwise be intractable to perform with current CFD methods. Future work should focus on investigations with comprehensive soot models developed for kerosene and the improvement of the newly developed ISRN method.

\section{ACKNOWLEDGMENTS}

This work was supported by the UK Engineering and Physical Sciences Research Council (EPSRC) and Rolls-Royce plc. Dr M. Sirignano and Prof. A. D’Anna from Università degli Studi di Napoli Federico II provided the soot sectional model used in this work. This is greatly acknowledged. The authors would also like to thank Prof. Hai Wang (Stanford University) and Prof. Fokion Egolfopoulos (University of Southern California) for the useful discussions regarding the HyChem mechanism.

\section{REFERENCES}

[1] D'Anna, Andrea. "Combustion-formed nanoparticles." Proceedings of the Combustion Institute Vol. 32, No. 1 (2009): pp. 593-613. DOI 10.1016/j.proci.2008.09.005.

[2] Rigopoulos, Stelios. "Modelling of Soot Aerosol Dynamics in Turbulent Flow." Flow, Turbulence and Combustion Vol. 103, No. 3 (2019): pp. 565-604. DOI 10.1007/s10494-01900054-8.

[3] Wang, Hai. "Formation of nascent soot and other condensed-phase materials in flames." Proceedings of the Combustion Institute Vol. 33, No. 1 (2011): pp. 41-67. DOI 10.1016/j.proci.2010.09.009.

[4] Rigopoulos, Stelios. "Population balance modelling of polydispersed particles in reactive flows." Progress in energy and combustion science Vol. 36, No. 4 (2010): pp. 412-443. DOI 10.1016/j.pecs.2009.12.001.

[5] Roy, Somesh P. and Haworth, Daniel C. "A Systematic Comparison of Detailed Soot Models and Gas-Phase Chemical Mechanisms in Laminar Premixed Flames." Combustion Science and Technology Vol. 188, No. 7 (2016): pp. 10211053. DOI 10.1080/00102202.2016.1145117.

[6] Chong, Shao Teng, Raman, Venkat, Mueller, Michael E., Selvaraj, Prabhu and Im, Hong G. "Effect of soot model, moment method, and chemical kinetics on soot formation in a model aircraft combustor." Proceedings of the Combustion Institute Vol. 37, No. 1 (2019): pp. 1065 - 1074. DOI 10.1016/j.proci.2018.06.093.

[7] Sewerin, Fabian and Rigopoulos, Stelios. "An LESPBE-PDF approach for predicting the soot particle size distribution in turbulent flames." Combustion and Flame Vol. 189 (2018): pp. 62-76. DOI 10.1016/j.combustflame.2017.09.045.

[8] Rodrigues, Pedro, Franzelli, Benedetta, Vicquelin, Ronan, Gicquel, Olivier and Darabiha, Nasser. "Coupling an LES approach and a soot sectional model for the study of sooting turbulent non-premixed flames." Combustion and Flame Vol. 190 (2018): pp. 477-499. DOI 10.1016/j.combustflame.2017.12.009.

[9] Zhang, Tongfeng, Zhao, Liyun, Kholghy, M. Reza, Thion, Sébastien and Thomson, Murray J. "Detailed investigation 
of soot formation from jet fuel in a diffusion flame with comprehensive and hybrid chemical mechanisms." Proceedings of the Combustion Institute Vol. 37, No. 2 (2019): pp. 2037-2045. DOI 10.1016/j.proci.2018.06.012.

[10] Gkantonas, Savvas, Sirignano, Mariano, Giusti, Andrea, D’Anna, Andrea and Mastorakos, Epaminondas. "Comprehensive Soot Particle Size Distribution Modelling Of A Model Rich-Quench-Lean Burner." 11th Mediterannean Combustion Symposium, Tenerife, Spain (2019). DOI 10.17863/CAM.43221.

[11] Gkantonas, Savvas, Sirignano, Mariano, Giusti, Andrea, D’Anna, Andrea and Mastorakos, Epaminondas. "Comprehensive soot particle size distribution modelling of a model Rich-Quench-Lean burner." Fuel Vol. 270 (2020): p. 117483. DOI 10.1016/j.fuel.2020.117483.

[12] Saffaripour, Meghdad, Kholghy, M. Reza, Dworkin, Seth B. and Thomson, Murray J. "A numerical and experimental study of soot formation in a laminar coflow diffusion flame of a Jet A-1 surrogate." Proceedings of the Combustion Institute Vol. 34, No. 1 (2013): pp. 1057-1065. DOI 10.1016/j.proci.2012.06.176.

[13] Smith, Nigel S.A. "Development of the conditional moment closure method for modelling turbulent combustion.” Ph.D. thesis, University of Sydney. 1994.

[14] Mobini, Kamran. "An investigation of the imperfectly stirred reactor modelling of recirculating combustion flows." Ph.D. thesis, University of Sydney. 1998.

[15] Mobini, Karman and Bilger, Robert W. "Imperfectly Stirred Reactor Model Predictions Of Reaction In A Burner With Strong Recirculation." Combustion Science and Technology Vol. 176, No. 1 (2004): pp. 45-70. DOI 10.1080/00102200490255334.

[16] Mobini, Karman and Bilger, Robert W. "Parametric study of the Incompletely Stirred Reactor modeling." Combustion and Flame Vol. 156, No. 9 (2009): pp. 1818-1827. DOI 10.1016/j.combustflame.2009.06.017.

[17] Klimenko, Alex Y and Bilger, Robert W. "Conditional moment closure for turbulent combustion." Progress in energy and combustion science Vol. 25, No. 6 (1999): pp. 595-687.

[18] Sitte, Michael Philip and Mastorakos, Epaminondas. "Large Eddy Simulation of a spray jet flame using Doubly Conditional Moment Closure." Combustion and Flame Vol. 199 (2019): pp. 309 - 323. DOI 10.1016/j.combustflame.2018.08.026.

[19] Gough, A.J., Mobini, Karman, Chen, Yungcheng and Bilger, Robert W. "Measurements and predictions in a confined bluff-body burner modeled as an imperfectly stirred reactor." Proceedings of the Combustion Institute Vol. 27, No. 2 (1998): pp. 3181-3188. DOI 10.1016/S00820784(98)80181-1.

[20] Attili, Antonio, Bisetti, Fabrizio, Mueller, Michael E. and Pitsch, Heinz. "Formation, growth, and transport of soot in a three-dimensional turbulent non-premixed jet flame." Combustion and Flame Vol. 161, No. 7 (2014): pp. 18491865. DOI 10.1016/j.combustflame.2014.01.008.
[21] Giusti, Andrea, Gkantonas, Savvas, Foale, Jenna M. and Mastorakos, Epaminondas. "Numerical Investigation of Flame Structure and Soot Formation in a Lab-Scale RichQuench-Lean Burner." Proceedings of the ASME Turbo Expo 2018: Turbomachinery Technical Conference and Exposition. Volume 4B: Combustion, Fuels, and Emissions. Turbo Expo: Power for Land, Sea, and Air: p. V04BT04A032. 2018. ASME, Oslo, Norway. DOI 10.1115/GT2018-76705.

[22] Lyra, Sgouria and Cant, Stewart. "Analysis of high pressure premixed flames using Equivalent Reactor Networks for predicting NOx emissions." Fuel Vol. 107 (2013): pp. 261 - 268. DOI 10.1016/j.fuel.2012.12.066.

[23] Giusti, Andrea, Mastorakos, Epaminondas, Hassa, Christoph, Heinze, Johannes, Magens, Eggert and Zedda, Marco. "Investigation of Flame Structure and Soot Formation in a Single Sector Model Combustor Using Experiments and Numerical Simulations Based on the Large Eddy Simulation/Conditional Moment Closure Approach." Journal of Engineering for Gas Turbines and Power Vol. 140, No. 6. DOI 10.1115/1.4038025.

[24] Wang, Hai, Xu, Rui, Wang, Kun, Bowman, Craig T., Hanson, Ronald K., Davidson, David F., Brezinsky, Kenneth and Egolfopoulos, Fokion N. "A physics-based approach to modeling real-fuel combustion chemistry - I. Evidence from experiments, and thermodynamic, chemical kinetic and statistical considerations." Combustion and Flame Vol. 193 (2018): pp. 502 - 519. DOI 10.1016/j.combustflame.2018.03.019.

[25] Xu, Rui, Wang, Kun, Banerjee, Sayak, Shao, Jiankun, Parise, Tom, Zhu, Yangye, Wang, Shengkai, Movaghar, Ashkan, Lee, Dong Joon, Zhao, Runhua, Han, Xu, Gao, Yang, Lu, Tianfeng, Brezinsky, Kenneth, Egolfopoulos, Fokion N., Davidson, David F., Hanson, Ronald K., Bowman, Craig T. and Wang, Hai. "A physics-based approach to modeling real-fuel combustion chemistry - II. Reaction kinetic models of jet and rocket fuels." Combustion and Flame Vol. 193 (2018): pp. 520 - 537. DOI 10.1016/j.combustflame.2018.03.021.

[26] Giusti, Andrea and Mastorakos, Epaminondas. "Turbulent Combustion Modelling and Experiments: Recent Trends and Developments." Flow, Turbulence and Combustion DOI 10.1007/s10494-019-00072-6.

[27] Garmory, Andrew and Mastorakos, Epaminondas. "Numerical simulation of oxy-fuel jet flames using unstructured LES-CMC." Proceedings of the Combustion Institute Vol. 35, No. 2 (2015): pp. 1207-1214. DOI 10.1016/j.proci.2014.05.032.

[28] Giusti, Andrea and Mastorakos, Epaminondas. "Detailed chemistry LES/CMC simulation of a swirling ethanol spray flame approaching blow-off." Proceedings of the Combustion Institute Vol. 36, No. 2 (2017): pp. 2625-2632. DOI 10.1016/j.proci.2016.06.035.

[29] Rigopoulos, Stelios and Jones, Alan. "A hybrid CFD_reaction engineering framework for multiphase reactor modelling: basic concept and application to bubble column reactors." Chemical Engineering Science Vol. 58, 
No. 14 (2003): pp. 3077-3089. DOI 10.1016/S00092509(03)00179-9.

[30] O'Brien, Edward E. and Jiang, Tai-Lun. “The conditional dissipation rate of an initially binary scalar in homogeneous turbulence." Physics of Fluids A: Fluid Dynamics Vol. 3, No. 12 (1991): pp. 3121-3123. DOI 10.1063/1.858127.

[31] Nehse, Maria, Warnatz, Jürgen and Chevalier, Christophe. "Kinetic modeling of the oxidation of large aliphatic hydrocarbons." Symposium (International) on Combustion Vol. 26, No. 1 (1996): pp. 773 - 780. DOI 10.1016/S00820784(96)80286-4.

[32] Dagaut, Philippe and Cathonnet, Michel. "The ignition, oxidation, and combustion of kerosene: A review of experimental and kinetic modeling." Progress in Energy and Combustion Science Vol. 32, No. 1 (2006): pp. 48 - 92. DOI 10.1016/j.pecs.2005.10.003.

[33] Wang, Hai, You, Xiaoqing, Joshi, Ameya V, Davis, Scott G, Laskin, Alexander, Egolfopoulos, Fokion and Law, CK. "USC Mech Version II. High-Temperature Combustion Reaction Model of H2/CO/C1-C4 Compounds." (2007).

[34] Wang, Yu, Raj, Abhijeet and Chung, Suk Ho. "A PAH growth mechanism and synergistic effect on PAH formation in counterflow diffusion flames." Combustion and Flame Vol. 160, No. 9 (2013): pp. 1667 - 1676. DOI 10.1016/j.combustflame.2013.03.013.

[35] Bolla, Michele, Wright, Yuri M., Boulouchos, Konstantinos, Borghesi, Giulio and Mastorakos, Epaminondas. "Soot Formation Modeling of n-Heptane Sprays Under Diesel Engine Conditions Using the Conditional Moment Closure Approach." Combustion Science and Technology Vol. 185, No. 5 (2013): pp. 766-793. DOI 10.1080/00102202.2012.752362.

[36] Leung, Kenneth M., Lindstedt, R. Peter and Jones, William P. "A simplified reaction mechanism for soot formation in nonpremixed flames." Combustion and Flame Vol. 87, No. 3 (1991): pp. 289 - 305. DOI 10.1016/00102180(91)90114-Q.

[37] Perini, Federico, Galligani, Emanuele and Reitz, Rolf D. "An Analytical Jacobian Approach to Sparse Reaction Kinetics for Computationally Efficient Combustion Modeling with Large Reaction Mechanisms." Energy \& Fuels Vol. 26, No. 8 (2012): pp. 4804-4822. DOI 10.1021/ef300747n.

[38] Brown, Peter N. and Hindmarsh, Alan C. "Reduced storage matrix methods in stiff ODE systems." Applied Mathematics and Computation Vol. 31 (1989): pp. 40-91. DOI 10.1016/0096-3003(89)90110-0.

[39] Sirignano, Mariano, Kent, John and D'Anna, Andrea. "Modeling formation and oxidation of soot in nonpremixed flames." Energy \& Fuels Vol. 27, No. 4 (2013): pp. 23032315.

[40] Wang, Yu and Chung, Suk Ho. "Soot formation in laminar counterflow flames." Progress in Energy and Combustion Science Vol. 74 (2019): pp. 152-238. DOI 10.1016/j.pecs.2019.05.003.

[41] Schneider, Denis, Meier, Ulrich, Quade, Wladislaw, Koopman, Johannes, Aumeier, Thomas, Langfeld, Andre,
Behrendt, Thomas, Hassa, Christoph and Rackwitz, Leif. "A new test rig for laser optical investigations of lean jet engine burners." 27th International Congress of the Aeronautical Sciences (ICAS 2010), Nice, France, September: pp. 19-24. 2010.

[42] Freitag, Stefan, Behrendt, Thomas, Heinze, Johannes, Lange, Lena, Meier, Ulrich, Rackwitz, L and Hassa, C. "Study of an airblast atomizer spray in a lean burn aeroengine model combustor at engine conditions." 24th European Conference on Liquid Atomization and Spray Systems (ILASS), Estoril, Portugal, Sept: pp. 5-7. 2011.

[43] Meier, Ulrich, Lange, L., Heinze, Johannes, Hassa, C., Sadig, S. and Luff, D. "Optical Methods for Studies of Self-Excited Oscillations and the Effect of Dampers in a High Pressure Single Sector Combustor." Journal of Engineering for Gas Turbines and Power Vol. 137, No. 7. DOI 10.1115/1.4029355. 072505.

[44] Lindstedt, R. Peter and Louloudi, Sofia A. "Joint-scalar transported PDF modeling of soot formation and oxidation." Proceedings of the Combustion Institute Vol. 30, No. 1 (2005): pp. 775-783. DOI 10.1016/j.proci.2004.08.080.

[45] El Helou, Ingrid, Skiba, Aaron W., Mastorakos, Epaminondas and Sidey, Jennifer A. "Investigation of the effect of dilution air on soot production and oxidation in a lab scale Rich-Quench-Lean (RQL) burner." AIAA Scitech 2019 Forum: p. 1436. 2019. DOI 10.2514/6.2019-1436.

[46] El Helou, Ingrid, Skiba, Aaron W. and Mastorakos, Epaminondas. "Investigation of soot production and oxidation in a lab-scale Rich-Quench-Lean (RQL) burner using in situ laser diagnostics." 11th Mediterannean Combustion Symposium, Tenerife, Spain (2019).

[47] El Helou, Ingrid, Skiba, Aaron W. and Mastorakos, Epaminondas. "Experimental Investigation of Soot Production and Oxidation in a Lab-Scale Rich-Quench-Lean (RQL) Burner." Flow, Turbulence and Combustion DOI 10.1007/s10494-020-00113-5.

[48] Gkantonas, Savvas, Giusti, Andrea and Mastorakos, Epaminondas. "Incompletely Stirred Reactor Network Modelling for Soot Emissions Prediction in Aero-Engine Combustors." International Workshop on Clean Combustion: Principles and Applications. 2019. AIAA, Darmstadt, Germany. DOI 10.17863/CAM.44973.

[49] Peter Geigle, Klaus, Hadef, Redjem and Meier, Wolfgang. "Soot Formation and Flame Characterization of an AeroEngine Model Combustor Burning Ethylene at Elevated Pressure." Journal of Engineering for Gas Turbines and Power Vol. 136, No. 2 (2013): pp. 021505-021505-7. DOI $10.1115 / 1.4025374$.

[50] Gkantonas, Savvas, Giusti, Andrea and Mastorakos, Epaminondas. "Incompletely Stirred Reactor Network Modeling of a Model Gas Turbine Combustor." AIAA Scitech 2020 Forum: p. 2087. 2020. DOI 10.2514/6.20202087.

[51] Chowdhury, Snehaunshu, Boyette, Wesley R. and Roberts, William L. "Time-averaged probability density functions of soot nanoparticles along the centerline of a piloted turbulent diffusion flame using a scanning mobility particle 
sizer.” J. Aerosol Sci. Vol. 106 (2017): pp. 56-67. DOI 10.1016/j.jaerosci.2016.10.012.

[52] De Falco, Gianluigi, El Helou, Ingrid, de Oliveira, Pedro M., Yuan, Ruoyang, D'Anna, Andrea and Mastorakos, Epaminondas (2019). Proceedings of the Combustion Institute,.

[53] Garmory, Andrew and Mastorakos, Epaminondas. "Capturing localised extinction in Sandia Flame F with LES-CMC." Proceedings of the Combustion Institute Vol. 33, No. 1 (2011): pp. 1673 -1680. DOI 10.1016/j.proci.2010.06.065.

\section{APPENDIX A. HYCHEM MODEL PAH EXTENSION}

In Fig. A1, the original HyChem mechanism is compared in $\eta$-space (using 0D-CMC as in Section 3.3) against the HyChem combined with additional PAH growth routes which form the base gas-phase scheme for the NAPS soot model. The additional reaction subset consists of 17 species and 43 reactions. The shared species and the temperature profiles have minor if any differences between the two mechanisms. Some of the additional aromatic species are shown, such as $\mathrm{A} 2 /$ naphthalene $\left(\mathrm{C}_{10} \mathrm{H}_{8}\right)$, A3/phenanphtrene $\left(\mathrm{C}_{14} \mathrm{H}_{10}\right)$, and $\mathrm{A} 4 /$ pyrene $\left(\mathrm{C}_{16} \mathrm{H}_{10}\right)$.

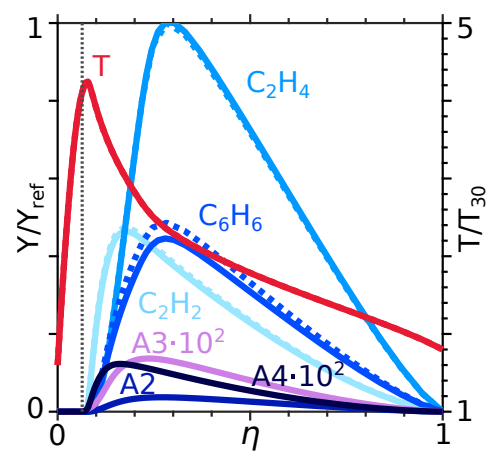

FIGURE A1: OD-CMC COMPARISON BETWEEN ORIGINAL HYCHEM (- - ) AND HYCHEM EXTENDED WITH PAH REACTION SUBSET (-) AT $N_{0}=101 / \mathrm{s}$ AND $p=1 \mathrm{bar}$ )

\section{APPENDIX B. CHOICE OF ISR SPACING}

The ultimate target of the ISRN design is to adequately resolve conditional gradients of reacting scalars, such as $\nabla Q_{\alpha}$ for a generic species, which appear on the LHS of Equation 6, either explicitly in the turbulent flux or implicitly as an absolute difference, $Q_{\alpha}-\left(Q_{\alpha}\right)_{j}$, in the inlet flux term. A good ISRN design ensures that transport effects are well captured, as also typically performed with conventional CMC methods where unsteady phenomena, e.g., extinction, are prominent [53]. Transport effects are especially important in soot evolution [2, 11, 44], therefore a more refined ISR spacing in soot production and oxidation regions can aid in improving predictability. Generally, a low number of ISRs is adequate to provide a quick estimate of soot emission, which is also the main advantage of the ISRN method: ensuring low computational costs. Nevertheless, a carefully selected ISR spacing may help optimize both computational efficiency and predictability by resolving well the most critical areas for soot evolution, while keeping the total number of ISRs low. An optimization method or a parametric analysis was out of the scope of this study and this will be the subject of future work. Here, the employed ISRN designs of Figs. 7a-7b were actually chosen arbitrarily with the total computational cost only in mind. A better rationale to choose the ISRN design, would be to control the extent of key controlling variables dictating soot evolution, namely the local equivalence ratio, scalar dissipation rate and residence time. In Fig. B1, the conditional scalar dissipation rate at stoichiometry is plotted against the respective local residence time, $\tau$, within the ISRs and colored by the FDF-averaged mixture fraction. The comparison shows the extent of the controlling variables and an indication of how smoothly they are changing from reactor to reactor. As can be seen for example in the case of the fine ISRN, most ISRs are found in a region of $\tau=0$ to $\tau=1$ $\mathrm{ms}$, where a wide range is observed but the density of fuel-rich ISRs is similar to fuel-lean ISRs. The latter ensures that rich regions in the flow are well represented, in contrast to the coarse ISRN where the majority of ISRs are fuel-lean. A problem with both spacings is clearly the extent of local residence time, so another consideration could be the adjustment of reactor volumes to ensure a more narrow distribution of residence times. In addition, the range of scalar dissipation rates (SDR) is important. Here, the values at stoichiometry are mostly concentrated around a value of $N_{\eta_{s t}^{* *}}^{*}=0.2 \mathrm{~s}^{-1}\left(N_{0} \approx 2 \mathrm{~s}^{-1}\right.$ assuming $\left.N_{\eta}^{* *}=N_{0} G_{\eta}\right)$, which generally favors soot growth. As a result, over-estimations of soot emission in certain regions, e.g., close to the fuel injector, may be a result of inadequate ISR spacing in regions of high mixture fraction gradients [23]. To sum up, considerations such as the ones described above can easily be employed to choose an appropriate spacing and provide physical meaning to ISRN design.

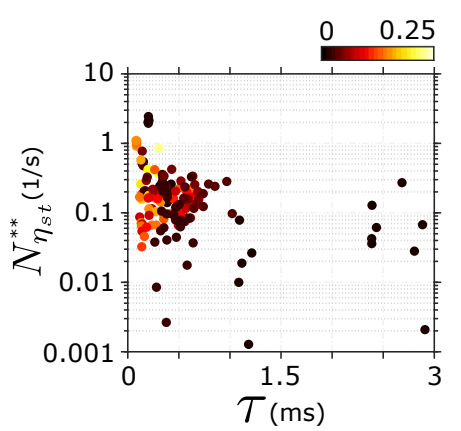

(a) Fine ISRN

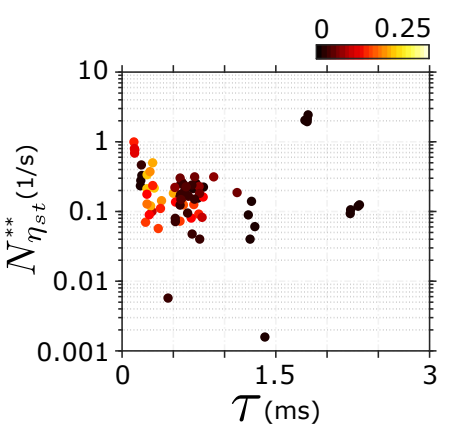

(b) Coarse ISRN
FIGURE B1: SCALAR DISSIPATION RATE AT STOICHIOMETRY $\left(N_{\eta_{s t}}^{* *}\right)$ VS. LOCAL REACTOR RESIDENCE TIME $(\tau)$ COLORED BY THE FDF-AVERAGED MIXTURE FRACTION 